\title{
Efeito da temperatura na cinética de \\ secagem em leito de espuma e na \\ degradação de antocianina em morango
}

\author{
Effect of temperature on drying kinetic in foam mat and \\ anthocyanin degradation in strawberry
}

\author{
Wallaf Costa Vimercati ${ }^{1 *}$ (D), Leandro Levate Macedo ${ }^{1}$ (D), Cintia da Silva Araújo1, \\ Luciano José Quintão Teixeira ${ }^{1}$, Sérgio Henriques Saraiva ${ }^{1}$
}

${ }^{1}$ Universidade Federal do Espírito Santo (UFES), Departamento de Engenharia de Alimentos, Alegre/ES - Brasil

${ }^{*}$ Corresponding Author: Wallaf Costa Vimercati, Universidade Federal do Espírito Santo (UFES), Centro de Ciências Agrárias e Engenharias, Departamento de Engenharia de Alimentos, Alto Universitário, s/n,

CEP: 29500-000, Alegre/ES - Brasil, e-mail: wallafcosta@hotmail.com

Cite as: Vimercati, W. C., Macedo, L. L., Araújo, C. S., Teixeira, L. J. Q., \& Saraiva, S. H. (2019). Effect of temperature on drying kinetic in foam mat and anthocyanin degradation in strawberry.. Brazilian Journal of Food Technology, 22, e2018221. https://doi.org/10.1590/1981-6723.22118

\begin{abstract}
Resumo
O morango é uma fruta de grande consumo no mundo e de grande riqueza nutricional, sendo utilizada como ingrediente na elaboração de diversos produtos. No entanto, trata-se de uma fruta de alta perecibilidade, exigindo tecnologias para o aumento da vida útil. Diante disso, o objetivo deste trabalho foi avaliar a cinética de secagem e o efeito da temperatura sobre o teor de antocianinas totais do morango. Para isso, a polpa do morango foi obtida e destinada à análise de antocianinas totais e umidade. A albumina a $1 \%$ foi adicionada à polpa e submetida à bateção para a elaboração da espuma. A espuma foi disposta em bandejas em secador convectivo nas temperaturas $55,60,65,70$ e $75^{\circ} \mathrm{C}$, até peso constante. Os modelos de Henderson e Pabis, Lewis e Page foram ajustados aos dados de razão de umidade. $O$ modelo generalizado da razão de umidade em função da temperatura e tempo de secagem foi construído. $O$ teor de antocianinas totais e a umidade dos pós foram determinados. $O$ modelo de Page foi escolhido para representar a cinética de secagem da polpa de morango, uma vez que apresentou o maior valor do coeficiente de determinação ajustado e o menor erro padrão da regressão. O modelo generalizado obtido foi satisfatório para prever a razão de umidade da polpa de morango, apresentando $R^{2}$ de 0.98 . $O$ teor de antocianinas totais da polpa in natura foi de $235,03 \mathrm{mg} / 100 \mathrm{~g}$, reduzindo aproximadamente $50 \%$ do conteúdo inicial para todas as temperaturas avaliadas.
\end{abstract}

Palavras-chave: Fragaria sp.; Pigmentos; Pelargonidina; Cinética de secagem; Modelagem matemática; Page.

\begin{abstract}
The strawberry is a fruit widely consumed throughout the world and of great nutritional wealth, being used as an ingredient in the elaboration of a variety of products. However, it is a highly perishable fruit, requiring technologies to increase its useful life. Therefore, the objective of this work was to evaluate the drying kinetics and the effect of temperature on the total anthocyanin content of the strawberry. Thus, strawberry pulp was obtained and destined for the analysis of the total anthocyanin and moisture contents. $1 \%$ albumin was added to the pulp and subjected
\end{abstract}


to beating to produce a foam. The foam was placed in trays in a convective dryer at temperatures of $55,60,65,70$ and $75{ }^{\circ} \mathrm{C}$, to constant weight. The Henderson and Pabis, Lewis and Page models were fitted to the moisture ratio data and the generalized model of the moisture ratio as a function of temperature and drying time constructed. The total anthocyanin and moisture contents of the powders were determined. The Page model was chosen to represent the drying kinetics of the strawberry pulp, since it presented the highest value for the adjusted coefficient of determination and the lowest standard error of the regression. The generalized model obtained was satisfactory to predict the moisture ratio of the strawberry pulp, presenting an $R^{2}$ of 0.98 . The total anthocyanin content of the fresh pulp was $235.03 \mathrm{mg} / 100 \mathrm{~g}$ and reduced by approximately $50 \%$ for all the temperatures evaluated.

Keywords: Fragaria sp.; Pigments; Pelargonidin; Drying kinetics; Mathematical modeling, Page.

\section{Introdução}

A fruticultura representa um dos setores mais importantes e representativos no mercado agrícola mundial, principalmente em países tropicais como o Brasil, onde as condições edafoclimáticas são favoráveis à produção de uma ampla gama de frutas. Tais características são responsáveis pela geração de empregos e têm contribuição significativa com o Produto Interno Bruto (PIB) de diversas regiões brasileiras. O impacto da fruticultura sobre comunidades agrícolas tem se tornado gradualmente relevante, visto que as frutas têm ganhado cada vez mais espaço na mesa dos consumidores (Moreira et al., 2013).

Mediante a busca do bem-estar físico e mental e a preocupação com a saúde, tem sido observada uma demanda crescente por produtos naturais e saudáveis. Dentro desse cenário, as frutas se destacam em consequência da riqueza e variedade de nutrientes, contendo, principalmente, as mais diversas vitaminas, minerais e compostos bioativos fundamentais à nutrição humana (Fachinello et al., 2011; Braga et al., 2018).

O morango (Fragaria sp.) é uma fruta delicada, frágil, não climatérica, de alta taxa respiratória, portanto, muito perecível e com vida útil de poucos dias (Malgarim et al., 2006). No Brasil, a produção de morango em 2017 foi de 3390 toneladas (Food and Agriculture Organization of the United Nations, 2018). A fruta in natura possui grande aceitação sensorial por boa parcela da população mundial. Esta fruta pode ainda ser destinada à formulação de diversos produtos, especialmente os alimentícios, tais como iogurtes, bebidas, panificados, doces e sorvetes (Oliveira et al., 2015; Castricini et al., 2017). Além do apreço sensorial, outro destaque do morango tem sido o valor nutricional e compostos bioativos. Em $100 \mathrm{~g}$ de morangos são encontrados vários minerais e vitaminas, tais como potássio $(153 \mathrm{~g})$, fósforo $(24 \mathrm{mg})$, cálcio $(16 \mathrm{mg})$, magnésio (13 mg), sódio (1 mg), ferro (0,41 mg), zinco (0,14 mg), vitaminas C (58,8 mg), B3 (0,386 mg), $\mathrm{E}(0,29 \mathrm{mg}), \mathrm{B} 6(0,047 \mathrm{mg}), \mathrm{B} 2(0,022 \mathrm{mg})$, folato $(24 \mu \mathrm{g}), \mathrm{K}(3,3 \mu \mathrm{g})$ e vitamina A $(1 \mu \mathrm{g})$ (United States Department of Agriculture, 2018).

As antocianinas são pigmentos naturais de tonalidade vermelho brilhante, pertencentes à família dos flavonoides, presentes em diversas frutas, flores, folhas, caules e raízes de plantas. Esses compostos apresentam atividade antioxidante, anticarcinogênica, antiviral e anti-inflamatória (Teixeira et al., 2008; Braga et al., 2018). A pelargonidina 3-O-glicosídeo, cianidina 3-glicosídeo e pelargonidina 3-rutinosídeo representam as principais antocianinas encontradas em morangos (Panico et al., 2009). Contudo, tem sido relatado que a pelargonidina 3-O-glicosídeo representa $90 \%$ das antocianinas totais do morango (Wojdyło et al., 2009). Aaby et al. (2012) relataram que a concentração de antocianinas em 27 cultivares de morango variou de $8.5 \mathrm{a} 65.9 \mathrm{mg} / 100 \mathrm{~g}$ de peso fresco.

Estudos desenvolvidos têm evidenciado o efeito benéfico do consumo de morangos na prevenção de doenças como problemas cardiovasculares, obesidade, síndrome metabólica, câncer e doenças neurológicas (Giampieri et al., 2015). 
Algumas frutas, em especial o morango, possuem sazonalidade, o que restringe a oferta e causa grande variabilidade no preço dessas frutas durante o ano. Além disso, podem apresentar alta perecibilidade, contribuindo com as perdas e desperdícios dos alimentos e com minimização do intercâmbio de frutas, principalmente as regionais, entre as diferentes localidades no mundo. Diante disso, métodos de conservação são essenciais para aumentar a vida útil, a disponibilidade e o consumo das frutas (Alves et al., 2010; Castricini et al., 2017).

A secagem é uma das opções para conservação de frutas, baseada na remoção de uma parcela da umidade do produto, reduzindo sua atividade de água e proporcionando maior estabilidade e vida útil. No entanto, a aplicação de calor pode provocar a degradação de uma série de compostos termolábeis, em especial as antocianinas (Casarin et al., 2016; Anandharamakrishnan, 2017; Teles et al., 2017).

A secagem em leito de espuma consiste na transformação do produto úmido em uma espuma, com o auxílio de agentes espumantes. A espuma proporciona o aumento da área de contato do produto com o ar de secagem, aumentando a taxa de secagem e, consequentemente, reduzindo o tempo de exposição ao calor. Dessa forma, o produto fica exposto a alta temperatura por menor tempo, minimizando a degradação de compostos termolábeis (Karim \& Wai, 1999; Kadam \& Balasubramanian, 2011; Araújo et al., 2017).

A facilidade de remoção de água durante a secagem é indicada pelo comportamento da curva. O estudo da cinética de secagem possibilita conhecer a temperatura apropriada e o tempo necessário para conclusão do processo. Além disso, a descrição efetiva por meio de equações matemáticas adequadas permite prever os parâmetros do processo a qualquer momento, a partir dos dados iniciais (Avhad \& Marchetti, 2016).

Nesse contexto, o objetivo deste trabalho foi avaliar a cinética de secagem em leito de espuma, bem como o efeito da temperatura de secagem sobre o teor de antocianinas totais do morango.

\section{Material e métodos}

\subsection{Planejamento experimental}

O experimento foi realizado em um delineamento inteiramente casualizado, com três repetições. As etapas de condução do experimento são apresentadas na Figura 1.

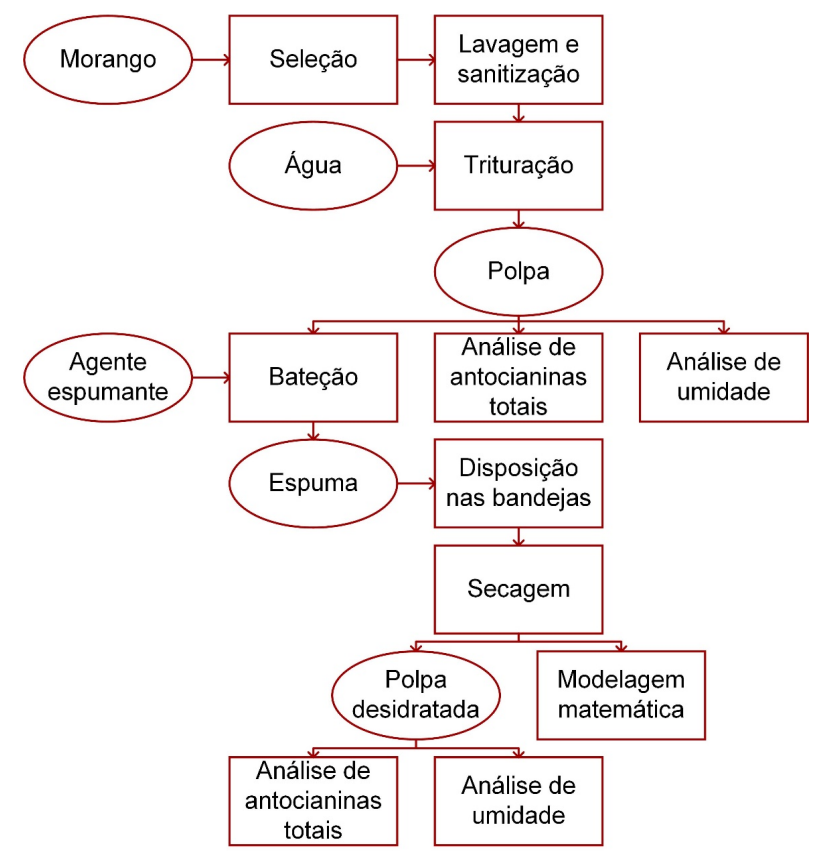

Figura 1. Etapas experimentais. 


\subsection{Obtenção da polpa e produção da espuma estável}

Os morangos foram selecionados, lavados e sanitizados com água clorada (200 ppm). A polpa dos frutos foi extraída em liquidificador doméstico, na proporção $(\mathrm{m} / \mathrm{v})$ de três partes de fruto para uma parte de água. Posteriormente, a mistura foi colocada em batedeira (ARNO planetária ${ }^{\circledR}$, modelo BPAI, $280 \mathrm{~W}, 50-60 \mathrm{~Hz}$ ) juntamente com $1 \%$ do agente espumante albumina, proporção determinada com base em estudos preliminares. Durante a produção da espuma, foi utilizada nos primeiros 5 minutos a velocidade mínima da batedeira, e nos 15 minutos restantes a velocidade máxima.

\subsection{Condução do processo de secagem}

As espumas produzidas foram dispostas em bandejas retangulares de aço inox, cujas dimensões são de $50 \times 40 \times 1 \mathrm{~cm}$. Posteriormente, as bandejas foram levadas ao secador convectivo com circulação forçada de ar $(1,5 \mathrm{~m} / \mathrm{s})$, nas temperaturas de $55,60,65,70$ e $75^{\circ} \mathrm{C}$. As massas das bandejas foram verificadas a cada 15 minutos durante a primeira hora, 30 minutos durante a segunda e terceira horas, e de hora em hora, até peso constante.

\subsection{Modelagem matemática}

A umidade durante a secagem foi expressa como razão de umidade (RU), conforme a Equação 1.

$R U=\frac{X_{t}-X_{e}}{X_{0}-X_{e}}=\frac{M_{t}-M_{e}}{M_{0}-M_{e}}$

em que, $X_{0}, X_{t}$ e $X_{e}$ são os valores da umidade, em base seca, da espuma úmida, no tempo $t$ horas da secagem e no equilíbrio; $M_{0}, M_{t}$ e $M_{e}$ são as massas, em gramas, da espuma úmida, no tempo thoras da secagem e no equilíbrio, respectivamente.

Aos dados experimentais da secagem foram ajustados os modelos de Henderson \& Pabis (1962), Lewis (1921) e Page (1949), conforme apresentado nas Equações 2 a 4, respectivamente. O programa SigmaPlot v.11.0 foi utilizado para ajuste dos modelos aos dados experimentais de RU, por meio de regressão não linear.

$$
\begin{aligned}
& R U=\mathrm{ae}^{-k t} \\
& \mathrm{RU}=\mathrm{e}^{-k t} \\
& \mathrm{RU}=\mathrm{e}^{-k \mathrm{kt}^{\mathrm{n}}}
\end{aligned}
$$

em que a, k e n são parâmetros dos modelos e té o tempo de secagem, em horas.

O grau de ajuste dos modelos foi comparado a partir dos valores do coeficiente de determinação ajustado $\left(\mathrm{R}^{2}\right.$ adj) e erro padrão da regressão (S), conforme apresentado nas Equações 5 e 6.

$$
\begin{aligned}
& \mathrm{R}_{\text {adj }}^{2}=1-\frac{\sum_{\mathrm{i}=1}^{\mathrm{N}}\left(\mathrm{RU}_{\text {exp }, \mathrm{i}}-\mathrm{RU}_{\text {prev }, \mathrm{i}}\right)^{2} \mathrm{~N}-1}{\sum_{\mathrm{i}=1}^{\mathrm{N}}\left(\mathrm{RU}_{\text {exp }, \mathrm{i}} \mathrm{RU}\right)^{2} \mathrm{~N}-\mathrm{p}} \\
& \mathrm{S}=\left(\frac{\sum_{\mathrm{i}=1}^{\mathrm{N}}\left(\mathrm{RU}_{\text {exp }, \mathrm{i}}-\mathrm{RU}_{\text {prev }, \mathrm{i}}\right)^{2}}{\mathrm{~N}-\mathrm{p}}\right)^{\frac{1}{2}}
\end{aligned}
$$

em que $R U_{\text {exp,i: }}$ : valores experimentais da razão de umidade para a i-ésima observação; $R U_{\text {prev, }, \text { : valores }}$ previstos da razão de umidade para a i-ésima observação; $\overline{\mathrm{RU}}$ é o valor médio da razão de umidade calculado a partir dos valores experimentais; $\mathrm{N}$ é o número de observações e p é o número de parâmetros no modelo. 


\subsection{Modelo generalizado}

A partir do modelo escolhido para representar o processo de secagem em leito de espuma da polpa de morango, um modelo generalizado foi proposto. Nesse caso, os parâmetros originais do modelo foram substituídos por funções que representavam, satisfatoriamente, o efeito da temperatura sobre os parâmetros originais.

\subsection{Análise de umidade}

A umidade foi determinada nas amostras de polpa in natura e nos pós pelo método em estufa, conforme determinado por Instituto Adolfo Lutz (2008).

\subsection{Determinação do teor de antocianinas totais}

As antocianinas totais foram determinadas na polpa in natura e nos pós, as quais foram extraídas com etanol 70\% acidificado com $\mathrm{HCl}$ a pH 2,0, conforme Francis (1982), sendo conduzida em temperatura de refrigeração e ao abrigo da luz. Para quantificação das antocianinas totais foi utilizado o método espectrofotométrico, conforme descrito por Rodriguez-Saona et al. (1998). O conteúdo de antocianinas totais foi expresso em pelargonidina 3-O-glicosídeo, cuja massa molar é de 433,2 $\mathrm{g} \mathrm{mol}^{-1}$. O coeficiente de absortividade molar utilizado foi de $31600 \mathrm{~L}^{-1} \mathrm{~cm}^{-1} \mathrm{~mol}^{-1}$, em comprimento de onda de $510 \mathrm{~nm}$.

A análise de variância (ANOVA) e o teste de Dunnett, ambos a 5\% de probabilidade, foram utilizados para comparação dos teores de antocianinas totais entre a polpa (controle) e os pós obtidos das diferentes temperaturas de secagem.

Os teores de antocianinas totais dos pós foram submetidos à ANOVA, a 5\% de probabilidade, e as médias foram comparadas entre si por intervalo de confiança ao nível de $95 \%$.

\section{Resultados e discussão}

\subsection{Modelagem matemática}

Os dados experimentais e os modelos ajustados de Henderson e Pabis, Page e Lewis são apresentados na Figura 2. 

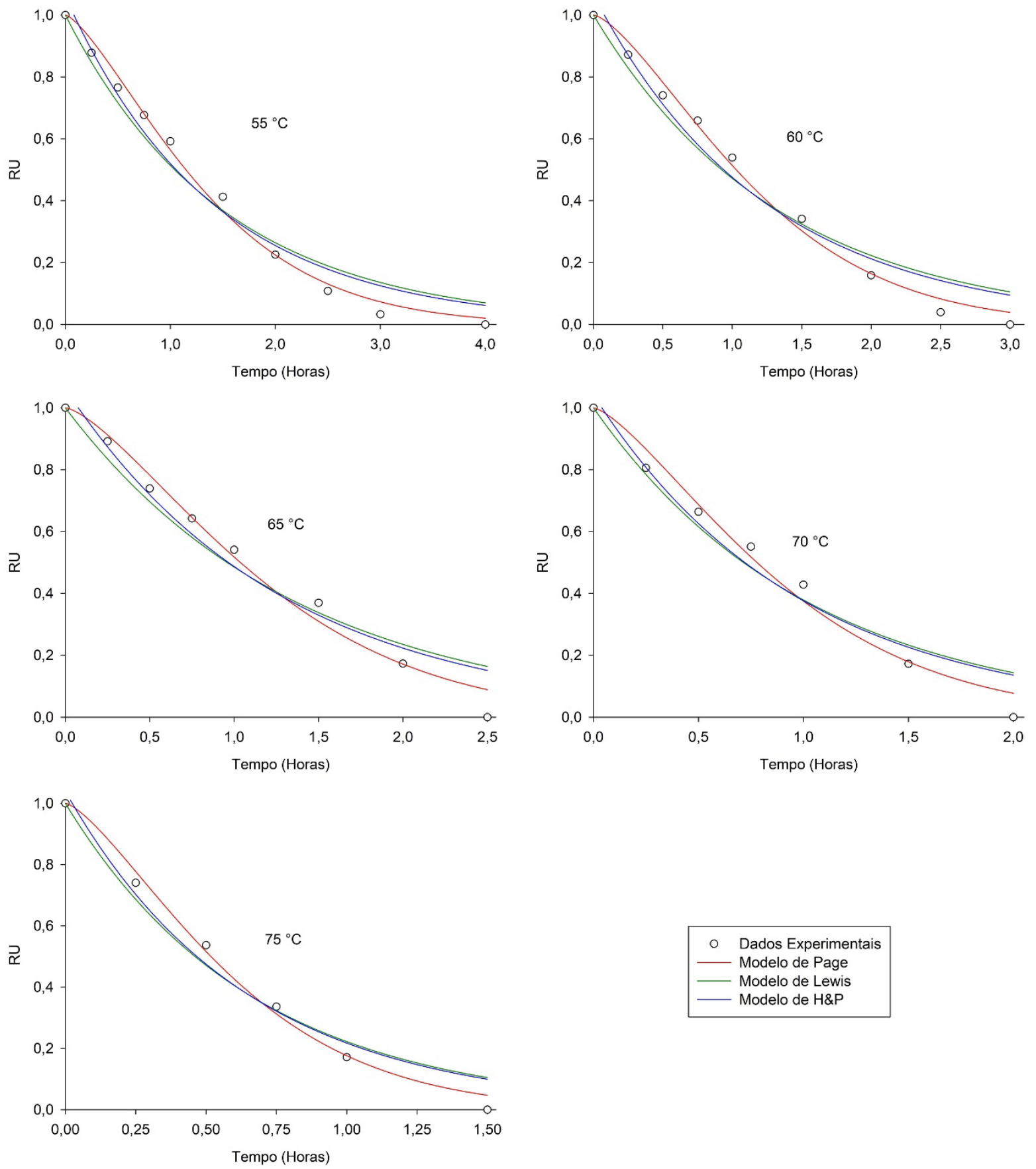

Figura 2. Curvas de secagem em leito de espuma da polpa de morango na faixa de temperatura de $55^{\circ} \mathrm{C}$ a $75^{\circ} \mathrm{C}$.

O tempo de secagem para as temperaturas de $75^{\circ} \mathrm{C}$ e $55^{\circ} \mathrm{C}$ foi de 1,5 e 4 horas, respectivamente, sendo 2,67 vezes menor para a maior temperatura. Com isso, foi verificado que o aumento da temperatura contribuiu para redução no tempo de secagem. Além disso, foi observado que a inclinação das curvas é mais acentuada quanto maior for a temperatura do ar de secagem, dada a maior transferência de calor do ar para o material a ser seco (Baptestini et al., 2015).

Os modelos matemáticos foram ajustados aos dados experimentais de razão de umidade ao longo do tempo de secagem da polpa de morango. Os valores dos parâmetros dos modelos, bem como o coeficiente de determinação ajustado ( $\mathrm{R}^{2}$ adj) e o erro padrão da regressão (S) são apresentados na Tabela 1. 
Tabela 1. Parâmetros dos modelos, valores de $\mathrm{R}^{2}$ adj e $\mathrm{S}$ da regressão.

\begin{tabular}{|c|c|c|c|c|}
\hline Temperatura $\left({ }^{\circ} \mathrm{C}\right)$ & Modelo & Modelo ajustado & $\mathbf{R}^{2}$ adj & $\mathbf{S}$ \\
\hline \multirow{3}{*}{55} & $\mathrm{H} \& \mathrm{P}$ & $\mathrm{RU}=1,0599 * \exp (-0,7122 * \mathrm{t})$ & 0,9691 & 0,0642 \\
\hline & Lewis & $\mathrm{RU}=\exp (-0,6656 * \mathrm{t})$ & 0,9668 & 0,0665 \\
\hline & Page & $\mathrm{RU}=\exp \left(-0,5727 *\left(\mathrm{t}^{1,3826}\right)\right)$ & 0,9920 & 0,0326 \\
\hline \multirow{3}{*}{60} & $\mathrm{H} \& \mathrm{P}$ & $\mathrm{RU}=1,0652 * \exp (-0,8068 * \mathrm{t})$ & 0,9596 & 0,0737 \\
\hline & Lewis & $\mathrm{RU}=\exp (-0,7506 * \mathrm{t})$ & 0,9576 & 0,0755 \\
\hline & Page & $\mathrm{RU}=\exp \left(-0,6663 *\left(\mathrm{t}^{1,4397}\right)\right)$ & 0,9901 & 0,0364 \\
\hline \multirow{3}{*}{65} & $\mathrm{H} \& \mathrm{P}$ & $\mathrm{RU}=1,0616 * \exp (-0,7795 * \mathrm{t})$ & 0,9487 & 0,0785 \\
\hline & Lewis & $\mathrm{RU}=\exp (-0,7234 * \mathrm{t})$ & 0,9481 & 0,0790 \\
\hline & Page & $\mathrm{RU}=\exp \left(-0,6585 *\left(\mathrm{t}^{1,4209}\right)\right)$ & 0,9802 & 0,0488 \\
\hline \multirow{3}{*}{70} & $\mathrm{H} \& \mathrm{P}$ & $\mathrm{RU}=1,0415 * \exp (-1,0184 * \mathrm{t})$ & 0,9483 & 0,0794 \\
\hline & Lewis & $\mathrm{RU}=\exp (-0,9706 * \mathrm{t})$ & 0,9534 & 0,0755 \\
\hline & Page & $\mathrm{RU}=\exp \left(-0,9803 *\left(\mathrm{t}^{1,3859}\right)\right)$ & 0,9768 & 0,0532 \\
\hline \multirow{3}{*}{75} & $\mathrm{H} \& \mathrm{P}$ & $\mathrm{RU}=1,0391 * \exp (-1,5647 * \mathrm{t})$ & 0,9654 & 0,0689 \\
\hline & Lewis & $\mathrm{RU}=\exp (-1,5044 * \mathrm{t})$ & 0,9695 & 0,0647 \\
\hline & Page & $\mathrm{RU}=\exp \left(-1,7363 *\left(\mathrm{t}^{1,3935}\right)\right)$ & 0,9916 & 0,0339 \\
\hline
\end{tabular}

Os modelos ajustados apresentaram altos valores de $\mathrm{R}^{2}$ adj $(\geq 0,94)$ e baixos valores de $\mathrm{S}(\leq 0,080)$ para todas as temperaturas avaliadas. Todavia, o modelo de Page foi o que apresentou o maior valor do coeficiente de determinação ajustado e o menor valor erro padrão da regressão, sendo, portanto, escolhido para representar o processo de secagem em leito de espuma da polpa de morango. Segundo Reis et al. (2013), valores de $\mathrm{S}$ menor que 1 indicam um bom ajuste do modelo. Outros autores, trabalhando com secagem em leito de espuma de caldo de cana (Marques et al., 2016) e acerola (Araújo et al., 2017) também verificaram que o modelo de Page foi o que melhor se ajustou aos dados experimentais.

\subsection{Modelo generalizado}

O modelo generalizado, conforme apresentado na Equação 7, é utilizado para estimar a razão de umidade de produtos desidratados em função da temperatura e tempo de secagem, simultaneamente.

$R U=\mathrm{e}^{\left[-\left(\mathrm{k} \mathrm{e}^{\frac{-k_{2}}{\mathrm{~T}}}\right) \mathrm{t}^{\mathrm{n}}\right]}$

Conforme observado na Equação 7, este modelo apresenta o fomato do modelo de Page. Os valores dos coeficientes de variação dos parâmetros $\mathrm{k}$ e $\mathrm{n}$ foram de $52,06 \%$ e 1,76\%, respectivamente. Com isso, foi notado que o parâmetro $\mathrm{k}$ do modelo de Page apresentou maior variação que o parâmetro $\mathrm{n}$. Para o parâmetro $\mathrm{k}$, foi verificada uma relação linear com o aumento da temperatura. Contudo, o parâmetro $\mathrm{n}$ não sofreu alteração com o aumento de temperatura, sendo considerado constante. 
Os valores estimados para os parâmetros do modelo generalizado são apresentados na Tabela 2.

Tabela 2. Estimativas dos parâmetros do modelo generalizado.

\begin{tabular}{cc}
\hline Parâmetro & Valor estimado \\
\hline $\mathrm{k}_{1}$ & $1,8275 \times 10^{7}\left(\mathrm{~h}^{-1}\right)$ \\
$\mathrm{k}_{2}$ & $5708,1441(\mathrm{~K})$ \\
$\mathrm{N}$ & 1,3985 \\
\hline
\end{tabular}

Os valores de RU predito versus experimentais pelo modelo generalizado são apresentados na Figura 3.

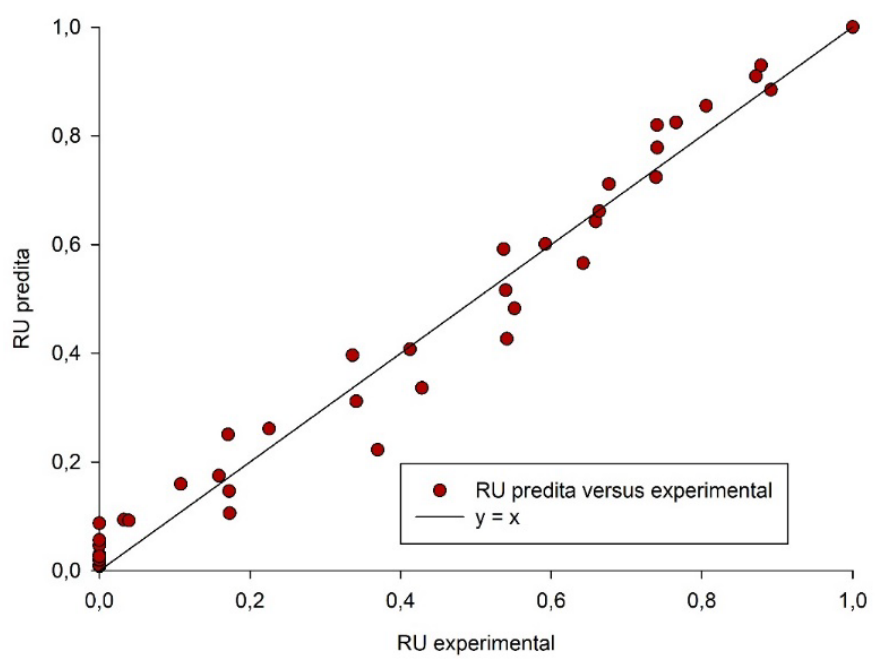

Figura 3. Valores de RU predita versus experimental.

O valor do coeficiente de determinação $\left(\mathrm{R}^{2}\right)$ do modelo generalizado foi de 0,98 , indicando alta proximidade entre os valores estimados pelo modelo generalizado com os dados experimentais.

\subsection{Análise de umidade}

A equação da razão de umidade (Equação 1) foi aplicada ao modelo generalizado (Equação 7). Posteriormente, a Equação 8 foi obtida.

$\mathrm{X}_{\mathrm{t}}=\mathrm{X}_{\mathrm{e}}+\left(\mathrm{X}_{0}-\mathrm{X}_{\mathrm{e}}\right) * \mathrm{e}^{\left[-\left(1,8275 * 10^{7} * \mathrm{e}^{-\frac{.5708,1441}{\mathrm{~T}}}\right) \mathrm{f}^{\mathrm{t}, 3985}\right]}$

em que, $\mathrm{X}_{0}, \mathrm{X}_{\mathrm{t}}, \mathrm{X}_{\mathrm{e}}$ são as umidades (\%), em base seca, nos tempos zero, t e equilíbrio, respectivamente; $T$ é a temperatura, em Kelvin; t é o tempo, em horas.

A umidade, em base úmida, da espuma da polpa de morango foi de 95,42\%. Por meio da Equação 9, foi obtida a umidade, em base seca, de 20,83 kg de água / kg de matéria seca.

$\mathrm{X}_{\mathrm{t}}=\frac{\mathrm{U}_{\mathrm{t}}}{100-\mathrm{U}_{\mathrm{t}}}$

em que, $X_{t}$ e $U_{t}$ são as umidades, em base seca, ( $\mathrm{kg}$ de água / $\mathrm{kg}$ de matéria seca) e úmida (\%), respectivamente.

A umidade de equilíbrio $\left(\mathrm{X}_{\mathrm{e}}\right)$ apresentou um decaimento exponencial com o aumento da temperatura, conforme observado na Figura 4. 


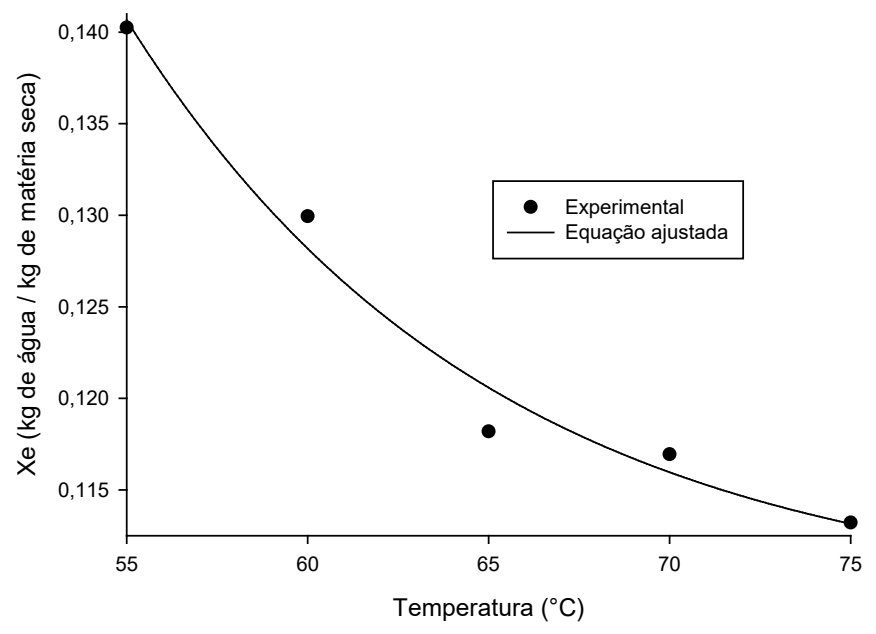

Figura 4. Umidade de equilíbrio versus temperatura.

O modelo matemático ajustado aos dados experimentais de umidade de equilíbrio em função da temperatura $\left({ }^{\circ} \mathrm{C}\right)$, Equação 10 , apresentou um bom ajuste aos dados experimentais, com coeficiente de determinação de 0,98 e erro padrão da regressão de 0,0022 .

$\mathrm{X}_{\mathrm{e}}=0,1088+7,5337 * \mathrm{e}^{(-0,09936 * \mathrm{~T})}$

Os valores de umidade no tempo zero e a equação da umidade de equilíbrio (Equação 10) foram aplicadas na Equação 8, obtendo-se a Equação 11.

$\mathrm{X}_{\mathrm{t}}=0,1088+7,5337 * \mathrm{e}^{(-0,09936 * \mathrm{~T})}+\left(20,8341-0,1088+7,5337 * \mathrm{e}^{(-0,09936 * \mathrm{~T})}\right) * \mathrm{e}^{\left[-\left(1,8275^{*} 10^{7 *} \mathrm{e}^{\frac{-5708,1441}{\mathrm{~T}}}\right) \mathrm{t}^{1,3985}\right]}$

A umidade da espuma, em base úmida, em função do tempo de secagem (h) e da temperatura do ar de secagem $\left({ }^{\circ} \mathrm{C}\right)$, foi obtida por meio das Equações 9 e 11. A superfície para a umidade é apresentada na Figura 5.

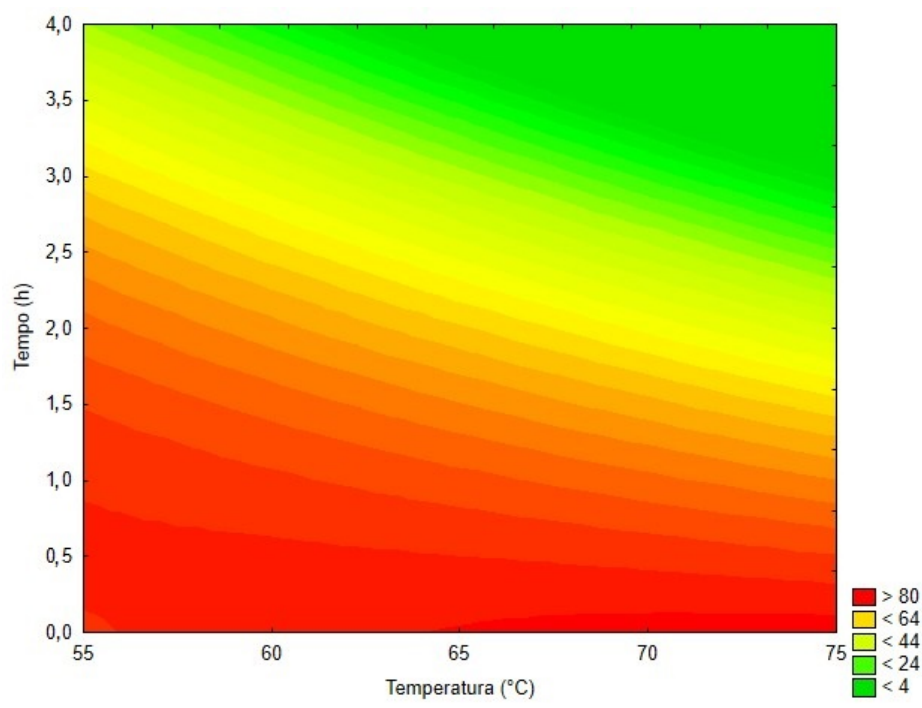

Figura 5. Umidade (\%), em base úmida, da espuma de morango em função da temperatura $\left({ }^{\circ} \mathrm{C}\right)$ e tempo (h) de secagem. 
A umidade da espuma reduziu com o aumento da temperatura e do tempo de secagem (Figura 5). Essas variáveis independentes influenciaram significativamente na umidade, conforme apresentado na Equação 12, com regressão significativa pelo teste $\mathrm{F}$ ao nível de $5 \%$ de probabilidade e coeficiente de determinação de 0,9811. Todos os parâmetros do modelo apresentaram-se significativos pelo teste t, ao nível de $5 \%$ de probabilidade. Sendo assim, o modelo ajustado foi capaz de representar a umidade da espuma de forma satisfatória, permitindo a estimativa dentro das condições específicas dos processos.

$\mathrm{U}_{\mathrm{t}}(\%)=5,1420+2,4798 * \mathrm{~T}-0,0116 * \mathrm{~T}^{2}+65,0728 * \mathrm{t}-4,9284 * \mathrm{t}^{2}-1,0929 * \mathrm{~T} * \mathrm{t}$

em que T é a temperatura, em Celsius $\left({ }^{\circ} \mathrm{C}\right)$, e t é o tempo, em horas.

\subsection{Determinação do teor de antocianinas totais}

A comparação dos teores médios de antocianinas da polpa (controle) entre as diferentes temperaturas (pós), em base seca, é apresentada na Figura 6A. Na Figura 6B, é apresentada a comparação dos teores médios de antocianina somente entre as temperaturas de secagem.
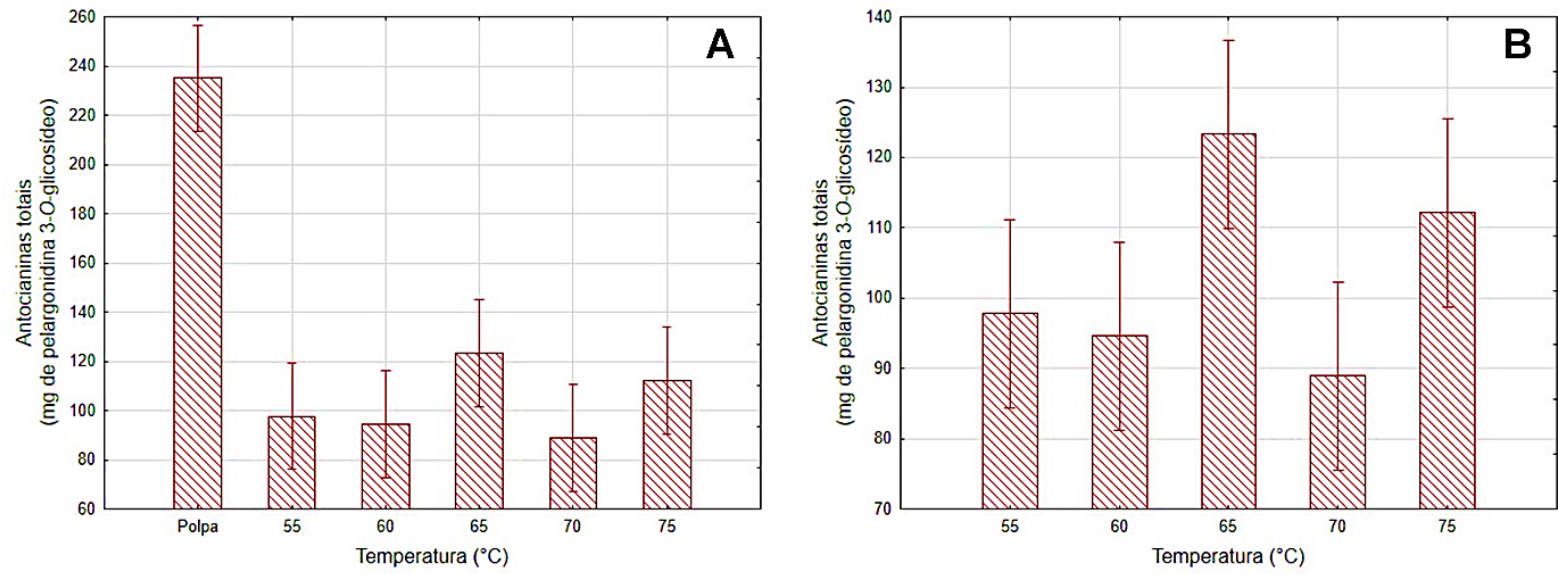

Figura 6. Médias e desvios-padrão de antocianinas da polpa e dos pós obtidos nas diferentes temperaturas de secagem. Comparações dos teores médios de antocianinas da polpa com as diferentes temperaturas de secagem, em base seca (A) e dos teores médios de antocianina somente entre as temperaturas de secagem, em base seca (B).

De acordo com a Figura 6A, o teor médio de antocianinas totais da polpa de morango in natura, expressa em pelargonidina 3-O-glicosídeo, foi de 235,03 mg / 100 gramas de amostra, em base seca. Valor próximo foi encontrado por Wojdyło et al. (2009), relatando um teor médio de $215,4 \mathrm{mg}$ deste constituinte por $100 \mathrm{~g}$ de morango, em base seca. Além disso, foi observado que o teor médio de pelargonidina na polpa in natura foi aproximadamente duas vezes maior que a média dos pós das diferentes temperaturas. A adição de albumina é responsável pelo aumento da matéria seca do produto. Entretanto, essa adição foi de apenas 1\%. Portanto, não se pode atribuir à albumina a ampla diferença entre o teor de antocianina, mas sim a exposição do produto às secagens.

$\mathrm{O}$ efeito das temperaturas de secagem pode ser observado na Figura $6 \mathrm{~B}$. A temperatura de $65{ }^{\circ} \mathrm{C}$ não diferiu estatisticamente das temperaturas de $55^{\circ} \mathrm{C} \mathrm{e} 75^{\circ} \mathrm{C}$. Uma provável explicação para esse fato é o efeito protetor que a albumina pode exercer sobre as antocianinas, conforme relatado por Chung et al. (2015). Além disso, pode ter ocorrido um efeito compensatório do tempo em relação à temperatura, levando a perdas semelhantes, ou seja, em temperaturas menores os tempos são maiores, sendo o contrário, também, verdadeiro. 


\section{Conclusão}

Os modelos matemáticos se ajustaram bem aos dados experimentais, com destaque para o modelo de Page, apresentando, para todas as temperaturas avaliadas, os maiores valores de coeficiente de determinação ajustado e menores valores do erro padrão da regressão.

A umidade do produto desidratado e o tempo de secagem da polpa de morango reduziram conforme o aumento da temperatura do ar.

O modelo generalizado foi adequado para estimar de forma satisfatória a razão de umidade da polpa de morango, permitindo o conhecimento da umidade do produto em função da temperatura e tempo de secagem.

A temperatura de secagem exerceu efeito significativo sobre o teor de antocianina do morango quando comparado com a polpa de morango in natura. Os níveis de temperatura avaliados não apresentaram uma relação direta com o teor de antocianina.

$\mathrm{O}$ teor de antocianina e de outros constituintes do morango podem ser estudados ao longo do processo de secagem em um maior intervalo de temperatura, assim como a avaliação da adição de biopolímeros que possam prolongar a estabilidade deste pigmento, de forma a contribuir com o avanço e otimização do processo de secagem.

\section{Agradecimentos}

À Fundação de Amparo à Pesquisa e Inovação do Espírito Santo (FAPES) pelas bolsas de Pesquisador Capixaba aos pesquisadores Sérgio Henriques Saraiva e Luciano José Quintão Teixeira. À Universidade Federal do Espírito Santo (UFES), pela oportunidade de realização deste trabalho.

\section{Referências}

Aaby, K., Mazur, S., Nes, A., \& Skrede, G. (2012). Phenolic compounds in strawberry (Fragaria x ananassa Duch.) fruits: Composition in 27 cultivars and changes during ripening. Food Chemistry, 132(1), 86-97. PMid:26434267. http://dx.doi.org/10.1016/j.foodchem.2011.10.037

Alves, J. A., Vilas Boas, E. V. B., Vilas Boas, B. M., \& Souza, É. C. (2010). Qualidade de produto minimamente processado à base de abóbora, cenoura, chuchu e mandioquinha-salsa. Food Science and Technology, 30(3), 625-634. http://dx.doi.org/10.1590/S0101-20612010000300009

Anandharamakrishnan, C. (2017). Handbook of drying for dairy products. New Jersey: John Wiley \& Sons. http://dx.doi.org/10.1002/9781118930526.

Araújo, C. S., Macedo, L. L., Vimercati, W. C., Saraiva, S. H., Oliveira, A. N., \& Teixeira, L. J. Q. (2017). Cinética de secagem de acerola em leito de espuma e ajuste de modelos matemáticos. Brazilian Journal of Food Technology, $20,1$. http://dx.doi.org/10.1590/1981-6723.15216

Avhad, M. R., \& Marchetti, J. M. (2016). Mathematical modelling of the drying kinetics of Hass avocado seeds. Industrial Crops and Products, 91, 76-87. http://dx.doi.org/10.1016/j.indcrop.2016.06.035

Baptestini, F. M., Corrêa, P. C., Junqueira, M. S., Ramos, A. M., Vanegas, J. D. B., \& Costa, C. F. (2015). Modelagem matemática da secagem de espuma de graviola. Revista Brasileira de Engenharia Agrícola e Ambiental, 19 (12), $1203-1208$. http://dx.doi.org/10.1590/1807-1929/agriambi.v19n12p1203-1208

Braga, A. R. C., Murador, D. C., De Souza Mesquita, L. M., \& De Rosso, V. V. (2018). Bioavailability of anthocyanins: Gaps in knowledge, challenges and future research. Journal of Food Composition and Analysis, 68, 31-40. http://dx.doi.org/10.1016/j.jfca.2017.07.031

Casarin, F., Mendes, C. E., Lopes, T. J., \& Moura, N. F. (2016). Planejamento experimental do processo de secagem da amora-preta (Rubus sp.) para a produção de farinha enriquecida com compostos bioativos. Brazilian Journal of Food Technology, 19, e2016025. http://dx.doi.org/10.1590/1981-6723.2516

Castricini, A., Dias, M. S. C., Martins, R. N., \& Santos, L. O. (2017). Morangos produzidos no semiárido de Minas Gerais: Qualidade do fruto e da polpa congelados. Brazilian Journal of Food Technology, 20. http://dx.doi.org/10.1590/19816723.14916

Chung, C., Rojanasasithara, T., Mutilangi, W., \& Mcclements, D. J. (2015). Enhanced stability of anthocyanin-based color in model beverage systems through whey protein isolate complexation. Food Research International, 76(Pt 3), 761-768. PMid:28455061. http://dx.doi.org/10.1016/j.foodres.2015.07.003 
Fachinello, J. C., Pasa, M. D. S., Schmtiz, J. D., \& Betemps, D. L. (2011). Situação e perspectivas da fruticultura de clima temperado no Brasil. Revista Brasileira de Fruticultura, 33(spe1), 109-120. http://dx.doi.org/10.1590/S010029452011000500014

Food and Agriculture Organization of the United Nations - FAOSTAT. (2018). Recuperado em 22 de dezembro de 2018, de http://www.fao.org/faostat/en/\#data/QC

Francis, F. J. (1982). Analysis of anthocyanins. In P. Markakis (Ed.), Anthocyanins as food colors (pp. 181-207). New York: Academic Press. http://dx.doi.org/10.1016/B978-0-12-472550-8.50011-1.

Giampieri, F., Forbes-Hernandez, T. Y., Gasparrini, M., Alvarez-Suarez, J. M., Afrin, S., Bompadre, S., Quiles, J. L., Mezzetti, B., \& Battino, M. (2015). Strawberry as a health promoter: An evidence based review. Food \& Function, 6(5), 1386-1398. PMid:25803191. http://dx.doi.org/10.1039/C5FO00147A

Henderson, S. M., \& Pabis, S. (1962). Grain drying theory I: Temperature effect on drying coefficient. Journal of Agricultural Research Engineering, 12, 732-736.

Instituto Adolfo Lutz - IAL. (2008). Métodos físicos-quimicos para análise de alimentos (4. ed.). São Paulo: IAL.

Kadam, D. M., \& Balasubramanian, S. (2011). Foam mat drying of tomato juice. Journal of Food Processing and Preservation, 35(4), 488-495. http://dx.doi.org/10.1111/j.1745-4549.2010.00492.x

Karim, A. A., \& Wai, C. C. (1999). Foam-mat drying of starfruit (Averrhoa carambola L.) puree: Stability and air drying characteristics. Food Chemistry, 64(3), 337-343. http://dx.doi.org/10.1016/S0308-8146(98)00119-8

Lewis, W. K. (1921). The rate of drying of solid materials. Journal of Industrial and Engineering Chemistry, 13(5), 427-432. http://dx.doi.org/10.1021/ie50137a021

Malgarim, M. B., Cantillano, R. F. F., \& Coutinho, E. F. (2006). Sistemas e condições de colheita e armazenamento na qualidade de morangos cv. Camarosa. Revista Brasileira de Fruticultura, 28(2), 185-189. http://dx.doi.org/10.1590/S010029452006000200007

Marques, G. M. R., Arruda, R. S., \& Silva, A. A. L. (2016). Secagem de caldo de cana em leito de espuma e avaliação sensorial do produto. Brazilian Journal of Food Research, 7(2), 16-29. http://dx.doi.org/10.3895/rebrapa.v7n2.3500

Moreira, T. B., Rocha, É. M. F. F., Afonso, M. R., \& Costa, J. M. C. (2013). Comportamento das isotermas de adsorção do pó da polpa de manga liofilizada. Revista Brasileira de Engenharia Agrícola e Ambiental, 17(10), 1093-1098

http://dx.doi.org/10.1590/S1415-43662013001000011

Oliveira, G. H. H., Aragão, D. M. S., Oliveira, A. P. L. R., Silva, M. G., \& Gusmão, A. C. A. (2015). Modelagem e propriedades termodinâmicas na secagem de morangos. Brazilian Journal of Food Technology, 18(4), 314-321.

http://dx.doi.org/10.1590/1981-6723.5315

Page, G. E. (1949). Factors influencing the maximum rates of air drying shelled corn in thin layers. West Lafayette: Purdue University.

Panico, A. M., Garufi, F., Nitto, S., Di Mauro, R., Longhitano, R. C., Magrì, G., Catalfo, A., Serrentino, M. E., \& De Guidi, G. (2009). Antioxidant activity and phenolic content of strawberry genotypes from fragaria $x$ ananassa. Pharmaceutical Biology, 47(3), 203-208. http://dx.doi.org/10.1080/13880200802462337

Reis, R. C., Côrrea, P. C., Devilla, I. A., Santos, E. S., Ascheri, D. P. R., Servulo, A. C. O., \& Souza, A. B. M. e. (2013). Drying of yam starch (Discorea ssp.) and glycerol filmogenic solutions at different temperatures. Lebensmittel-Wissenschaft + Technologie, 50(2), 651-656. http://dx.doi.org/10.1016/j.Iwt.2012.07.033

Rodriguez-Saona, L. E., Giusti, M. M., \& Wrolstad, R. E. (1998). Anthocyanin pigment composition of red-fleshed potatoes. Journal of Food Science, 63(3), 458-465. http://dx.doi.org/10.1111/j.1365-2621.1998.tb15764.x

Teixeira, L. N., Stringheta, P. C., \& Oliveira, F. A. (2008). Comparação de métodos para quantificação de antocianinas. Revista Ceres, 55(4), 297-304.

Teles, A. S. C., Chávez, D. W. H., Gomes, F. S., Cabral, L. M. C., \& Tonon, R. V. (2017). Effect of temperature on the degradation of bioactive compounds of Pinot Noir grape pomace during drying. Brazilian Journal of Food Technology, 21, e2017059. http://dx.doi.org/10.1590/1981-6723.5917

United States Department of Agriculture - USDA. Agricultural Research Service. National Nutrient Database for Standard Reference Legacy Release. (2018). Version Current: April. Recuperado em 20 de dezembro de 2018, de https://ndb.nal.usda.gov/ndb/foods/show/09316

Wojdyło, A., Figiel, A., \& Oszmiański, J. (2009). Effect of drying methods with the application of vacuum microwaves on the bioactive compounds, color, and antioxidant activity of strawberry fruits. Journal of Agricultural and Food Chemistry, 57(4), 1337-1343. PMid:19170638. http://dx.doi.org/10.1021/jf802507j

Funding: Fundação de Amparo à Pesquisa e Inovação do Espírito Santo (FAPES) pelas bolsas de Pesquisador Capixaba aos pesquisadores Sérgio Henriques Saraiva e Luciano José Quintão Teixeira (Projeto: 04/2015). 